\title{
Endovascular Stenting for Traumatic Common Iliac Pseudoaneurysm as an Adjunct following Exploratory Laparotomy in a Stab Injury Patient
}

\author{
${ }^{1}$ Saptarshi Biswas, ${ }^{2}$ Nishaan Nagarakanti
}

\begin{abstract}
Traumatic iliac vessel injuries, especially secondary to penetrating wounds, are often associated with a very high mortality rate. The patients usually present to the trauma bay in profound hemorrhagic shock, which, in turn, triggers the vicious cycle of hypothermia, coagulopathy, and acidosis. Iliac artery injuries should be managed promptly because of the high incidence of late complications of pseudoaneurysm and arteriovenous fistulae and risks of subsequent arterial thrombosis and distal ischemia.

We report a case of a 17-year-old female who was brought in following a stab wound in the right lower quadrant (RLQ). A postlaparotomy computed tomography (CT) scan performed revealed a pseudoaneurysm on the right common iliac. A pelvic angiography was performed, which was followed by an endovascular placement of a covered stent on the right common iliac artery.

We discuss an interesting case along with discussion of relevant literature.
\end{abstract}

Keywords: Common iliac artery, Pseudoaneurysm, Stab wound, Stenting.

How to cite this article: Biswas S, Nagarakanti N. Endovascular Stenting for Traumatic Common Iliac Pseudoaneurysm as an Adjunct following Exploratory Laparotomy in a Stab Injury Patient. Panam J Trauma Crit Care Emerg Surg 2016;5(3):172-176.

Source of support: Nil

Conflict of interest: None

\section{INTRODUCTION}

Traumatic iliac vessel injuries, especially secondary to penetrating wounds, are often associated with high mortality rates. The patients usually present with shock, due to massive blood loss, which, in turn, triggers the vicious cycle of hypothermia, coagulopathy, and acidosis.

We report the case of a 17-year-old female who was brought in with a stab wound in the right lower

\footnotetext{
${ }^{1}$ Attending Surgeon, ${ }^{2}$ Student

1,2Department of Trauma and Acute Care Surgery, Forbes Regional Hospital, Allegheny Health Network, Monroeville Pennsylvania, USA

Corresponding Author: Saptarshi Biswas, Attending Surgeon Department of Trauma and Acute Care Surgery, Forbes Regional Hospital, Allegheny Health Network, Monroeville, Pennsylvania USA, Phone: +4128582878, e-mail: saptarshibiswas@comcast. net
}

quadrant (RLQ). On exploratory laparotomy, there was a duodenal perforation with a retroperitoneal hematoma along the right Gerota's fascia and right colon. However, medial rotation of the right-sided viscera did not reveal any obvious injury to the inferior vena cava and the iliac veins. After stabilizing the patient, the computed tomography (CT) scan performed postlaparotomy revealed a pseudoaneurysm on the right common iliac artery. A pelvic angiography was performed, which was followed by an endovascular placement of a covered stent on the right common iliac artery.

We discuss an interesting case along with discussion of relevant literature.

\section{CASE REPORT}

A 17-year-old female patient was brought in as an activated level 1 trauma following a stab wound in the RLQ. On primary survey, the patient was awake, but she was lethargic and barely responded to commands. Vitals on presentation to the trauma bay were as follows: Heart rate $145 \mathrm{bpm}$, respiration rate 30 , and blood pressure at 101/70 saturating $99 \%$ on nonrebreather mask. She was started on $2 \mathrm{~L}$ of crystalloids followed by 4 units of O-negative blood. A massive transfusion protocol was then initiated for the patient.

A chest X-ray (Fig. 1) was obtained, which was viewed as negative for a pneumothorax. The patient was taken

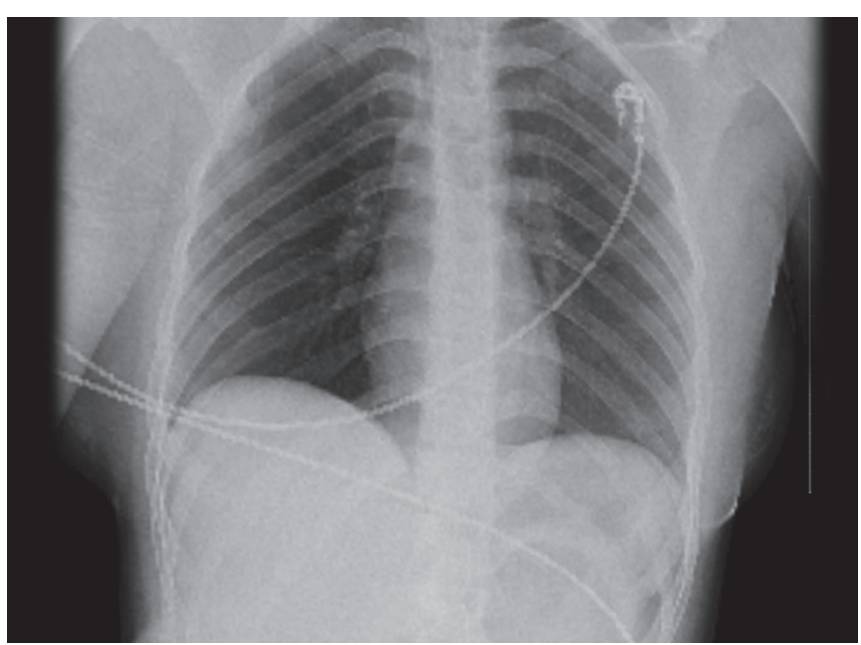

Fig. 1: Chest $\mathrm{X}$-ray in the trauma bay 


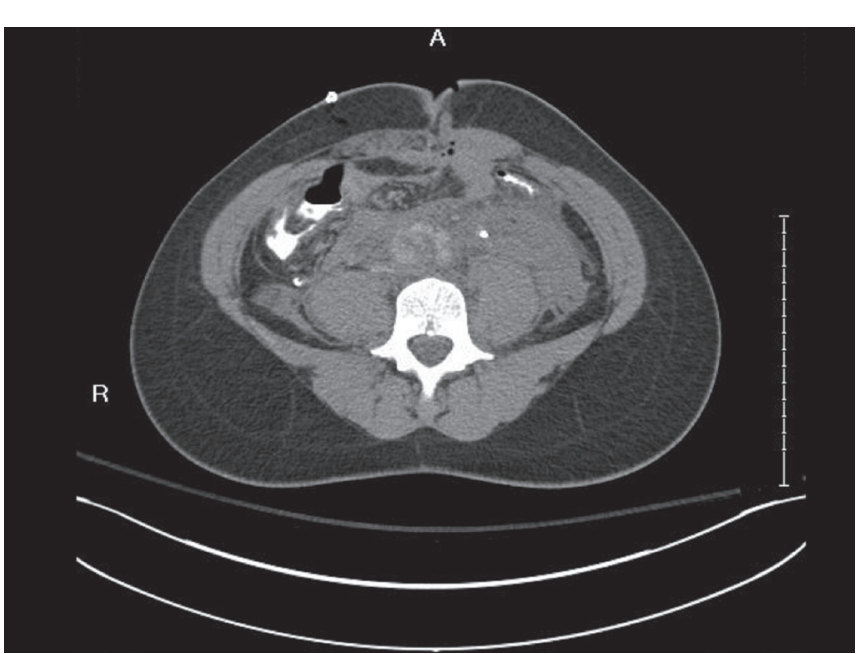

Fig. 2: Postoperative CT scan showing retroperitoneal hematoma

emergently to the operating room where she was intubated and underwent exploratory laparotomy. During the laparotomy, besides routine evaluation of solid organ injuries, a Cattell Braasch maneuver was performed to evaluate the right-sided organs and vessels to rule out retroperitoneal injuries. A perforation on the anterior surface of the duodenum was discovered. The duodenal perforation was closed primarily with interrupted sutures. A tongue of omentum was fashioned to reinforce the perforation. Medial rotation of the right-sided viscera showed a nonexpanding retroperitoneal hematoma, a right psoas muscle injury with some muscular bleeding, and a hematoma surrounding Gerota's fascia of the right kidney. No obvious injury to the iliac vessels was identified.

The patient was then transferred to the surgical intensive care unit (ICU) for further volume resuscitation and correction of coagulopathy, hypothermia, and the base deficit.

A CT scan of the chest, abdomen, and pelvis (Fig. 2) was performed with intravenous (IV) contrast. A delayed

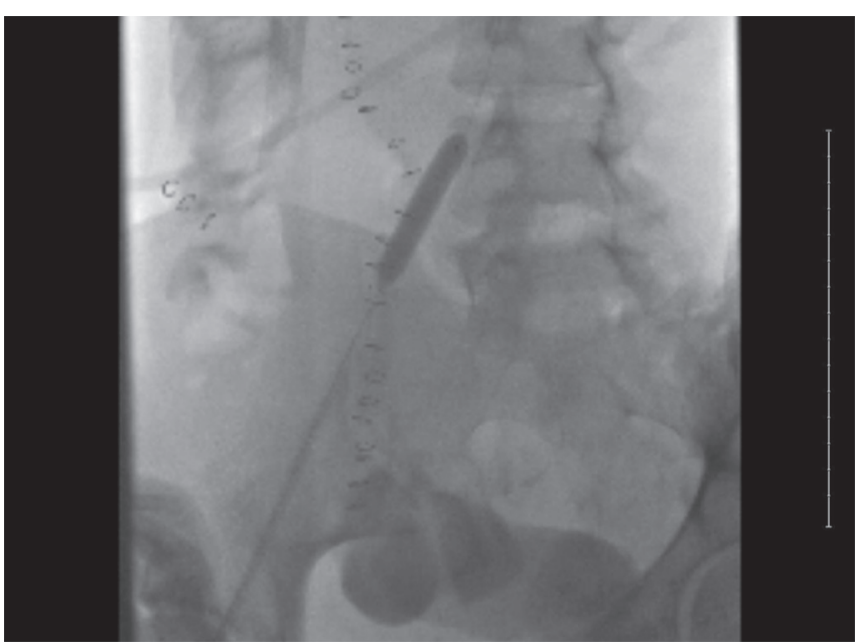

Fig. 4: Sheath placement

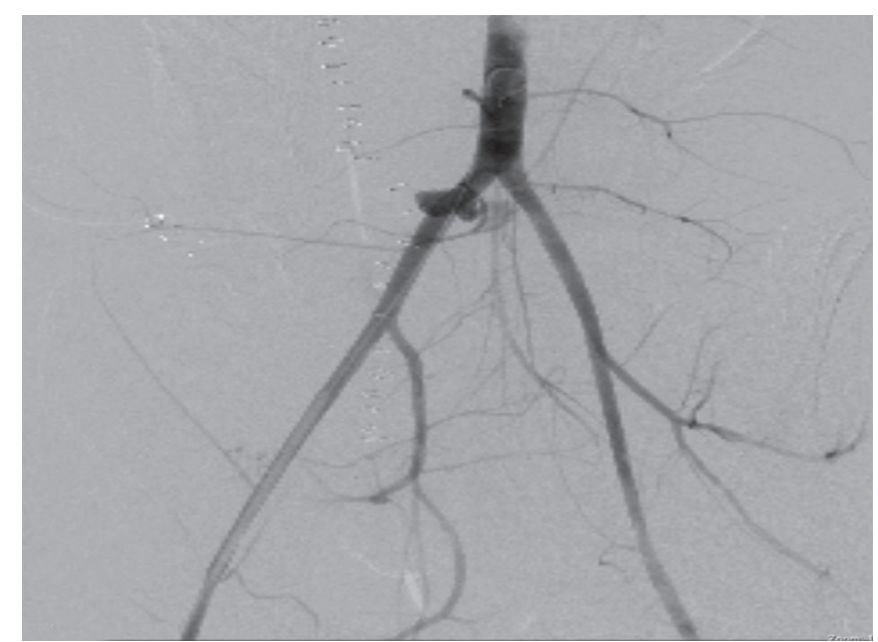

Fig. 3: Common iliac artery (CIA) pseudoaneurysm

phase scan of the pelvis was obtained to evaluate possible bladder injury. The CT showed a 5-mm pseudoaneurysm at the right common iliac artery (Fig. 3). It also showed intraperitoneal fluid, pneumoperitoneum secondary to the recent laparotomy, and a duodenal injury consistent with the residual bowel injury.

A pelvic angiography with covered stent placement on the right common iliac artery was done by interventional radiology, due to the moderate-sized pseudoaneurysm shown on the CT. Under concurrent ultrasound guidance, the micropuncture technique was utilized to access the right common femoral artery. The access was upsized to allow placement of a standard guide wire, followed by a 6 Fr sheath (Fig. 4). An Omni Flush catheter was positioned within the distal abdominal aorta and the left and right anterior oblique projection was used to better define the iliac bifurcation. A V-18 0.018 wire was then inserted and passed into the midabdominal aorta (Fig. 5). Over this, a $8 \mathrm{~mm} \times 5 \mathrm{~cm}$ VIABAHN stent was advanced utilizing a Fluoro Fade technology. The stent was successfully

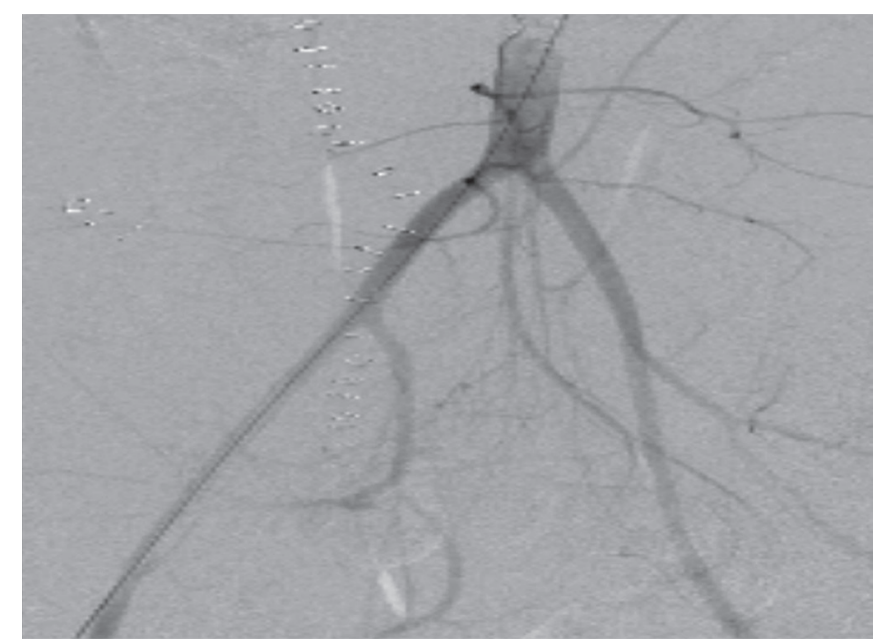

Fig. 5: Guide wire prior to stent placement 


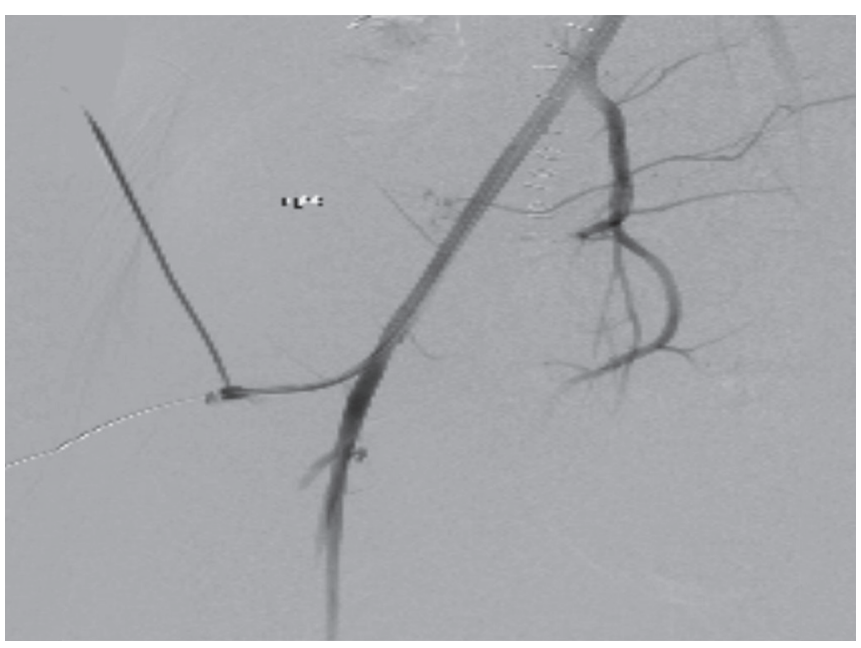

Fig. 6: Poststent placement

positioned at the origin of the right common iliac artery. The stent was then dilated utilizing an $8 \mathrm{~mm} \times 4 \mathrm{~cm}$ EverCross balloon. The Omni Flush catheter was once again positioned in the distal abdominal aorta and follow-up imaging was obtained. The stent appeared well apposed to the vessel wall, and there was no further filling of the pseudoaneurysm. Digital subtraction angiography imaging was then performed at the right groin puncture site. The site was deemed suitable for the placement of a closure device. A 7 Fr Mynx device (Fig. 6) was successfully deployed and manual pressure was held until hemostasis was achieved. The patient was returned to the ICU in stable condition.

On postoperative day 3 , a simple contrast watersoluble upper gastrointestinal follow-through (Fig. 7) was performed to rule out a leak of the repaired duodenum. There was no evidence of extraluminal contrast to suggest a leak. There, however, was a light delay of progression of contrast from the duodenum to the proximal jejunum. This was most likely due to a mild amount of postoperative edema.

A pulmonary embolism (PE) protocol CT scan was performed on postoperative day 6 for persistent tachycardia. Although negative for PE, new small bilateral pleural effusions were found besides adjacent compressive atelectasis. The CT revealed that the retroperitoneal hematoma decreased slightly. There was also mild bilateral renal collecting system fullness present. This was most likely due to the compression of the ureter by the retroperitoneal hematoma. The findings of the pelvis showed that the vascular stent across the right common iliac artery appeared to be patent. The extraperitoneal hematoma of the pelvis also showed slight decrease in volume.

On postoperative day $15, \mathrm{a} C \mathrm{~T}$ of the abdomen-pelvis was ordered to rule out a possible abscess as workup for pyrexia and diarrhea. The CT came back negative for an abscess. A magnetic resonance cholangiopancreatography

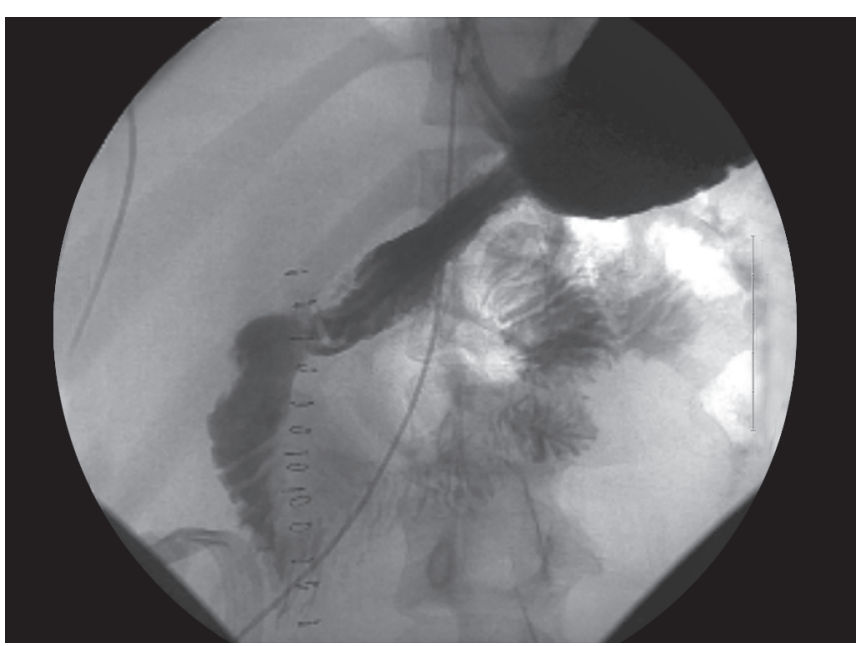

Fig. 7: Flow of contrast into the jejunum

(MRCP) was ordered the next day, without IV contrast, to see if there was an injury to the biliary system or pancreas. This was important considering its vicinity to the injured duodenum. The results of the MRCP were unremarkable and showed that the retroperitoneal hematoma was unchanged.

The patient made an unremarkable recovery and was discharged on postoperative day 17. She was followed up as an outpatient in a week's time, and she was afebrile, vitals stable, pain adequately controlled, and tolerating oral diet. We followed the patient in the clinic with a thorough clinical examination along with a scheduled ultrasonography (USG) at 4 weeks and a repeat CT at 3 months. The patient was lost to further follow-up.

\section{DISCUSSION}

The finding of the pseudoaneurysm at the right common iliac artery was incidental on the CT scan of the abdomen and pelvis. Isolated traumatic aneurysms of the common iliac artery are very rare. Although it is estimated that the prevalence of this type of aneurysm in vascular literature is between 0.008 and $0.03 \%,{ }^{1}$ the true incidence in trauma literature is difficult to predict considering its rarity and description in isolated case reports. About half of the patients are asymptomatic ${ }^{2}$ (as in our case report) and often very hard to identify through physical examination. The other half of the patients may present with symptoms based on the structure the aneurysm is compressing. These can manifest as hydroureter or deep venous thrombosis. ${ }^{3}$ The cause of mortality is most commonly attributed to the rupture of the aneurysm.

The risk of rupture increases with the size of the aneurysm itself. There is a 31\% rate of rupture in aneurysms with a mean diameter of $5.6 \mathrm{~cm} .{ }^{1}$ It is suggested that an asymptomatic aneurysm, i.e., greater than $3 \mathrm{~cm}$ diameter needs to be addressed with surgery. Other indications for repair are a ruptured iliac artery aneurysm, 
if it is symptomatic, if it is rapidly expanding $(>7 \mathrm{~mm}$ in 6 months or $>1 \mathrm{~cm}$ in 1 year), or if there is a coexisting aortic abdominal aneurysm repair. ${ }^{3}$ However, the statistics are scant in trauma literature.

A pseudoaneurysm or false aneurysm, by definition, is not bound by the three layers of the vessel wall. It is a hematoma that contains the break of the vessel that blood leaks through caused by trauma to the arterial vessels. In penetrating trauma, as in our case, though a rare complication, it should be suspected in a lower abdominal stab injury.

In this case, the patient underwent a CT scan with IV contrast for further testing. It is recommended when there is penetrating trauma to the abdomen, a CT scan with contrast may be performed if hemodynamically stable. As stated above, the CT revealed a 5-mm pseudoaneurysm of the right common iliac artery. Most aneurysms that are found in such scenarios are an incidental finding. There are different ways to diagnose a pseudoaneurysm. Using a CT angiography is a way to image arterial vessels and can help determine the exact location as in our case. The duplex USG can also be used.

The CT scans are routinely used to identify injuries in hemodynamically stable trauma patients. ${ }^{4}$ However, patients who are hemodynamically unstable and have definite indications for emergent/urgent surgery, secondary to penetrating or blunt trauma to the abdomen, undergo exploratory laparotomy. The CT scan is followed up soon after. The CTs are useful in picking up unsuspecting injuries. More vascular injuries are found in the setting of penetrating trauma, ${ }^{4-6}$ which can be partly because of the increased incidence in penetrating trauma. The CT scans are credited to identify injuries that are suboptimally assessed during the preceding exploration, often due to hemodynamic instability, thus requiring damage control strategy. Thorough exploration of the deep pelvis can also be challenging at times. Weis ${ }^{7}$ reported in his cohort of postlaparotomy CT scan that $7.2 \%$ of patients required additional surgery or angiography, while Haste et $\mathrm{al}^{8}$ in a separate retrospective study came up with a higher $8.9 \%$. Thus, postlaparotomy CT scan is a valuable adjunct to emergency surgery in trauma setting, often depicting unexpected injuries, besides confirming the presence of suspected injuries that were not fully explored at initial surgery. ${ }^{8}$

The decision of whether to use open surgical technique or endovascular repair is based on what the surgeon believes is best in that particular scenario. Conventionally, open repair was used in the past for almost all iliac artery aneurysms. Today, the paradigm has shifted to endovascular management in most institutions including ours. There are advantages of using endovascular repair. The major advantage is that it is a minimally invasive procedure.
The length of hospital stay is shorter. ${ }^{9}$ There is also less time in the operating room, which lowers morbidity, ${ }^{9}$ especially in a polytrauma elderly patient with multiple comorbidities. Furthermore, there is less blood loss and less need for transfusion. ${ }^{10}$ It also reduces the risks of open surgery. With open pelvic surgery, there is a risk of damaging sympathetic and parasympathetic nerves. In addition, lymph channels can be damaged resulting in a leak. If patients have had surgery in the past, the recovery from another open surgery can be challenging. In certain special cases, the procedure can also be done with local anesthesia and sedation instead of using general anesthesia, thus reducing overall perioperative morbidity. It is also found that mortality is lower in patients that go with the endovascular approach. However, this can also be due to the fact that open repair is undertaken for more severe hemodynamically unstable cases.

The disadvantages of this procedure are the need for IV contrast and further imaging. ${ }^{2}$ The use of IV contrast is nephrotoxic and can cause damage to the compromised kidneys resulting in acute kidney injury. Of course, the more imaging, i.e., done on a patient, the more chances that there can be complications from radiation. It is advised to use open repair for ruptures that will result in hemodynamic instability. It is also better for decompression of the aneurysm. ${ }^{2}$

As with any procedure, there are complications. The chance of perioperative morbidity is 10 to $23 \% .{ }^{11}$ Endoleak is the most common complication with endovascular repair. It occurs in 0 to $7 \%$ of patients. ${ }^{2}$ Endoleak can lead to rupture in $40 \%$ of the patients. ${ }^{2}$ Again, these statistics are based on vascular literature, as incidence of traumatic iliac psudoaneurysms is scarce. The femoral artery access site can potentially become infected. Hematomas and pseudoaneurysms can also form around the area. The stent itself can have issues. Thrombosis of the stent-graft can occur right after the procedure or during the weeks after. ${ }^{12}$ Anticoagulation should be started on patients to avoid this complication. If the graft does become occluded, another procedure must be done to resolve the problem, such as a femoro-femoral crossover bypass or transcatheter thrombolysis. ${ }^{13}$ Buttock claudication, though relatively rare, is another complication of endovascular repair. Usually, claudication after endovascular repair resolves on its own. ${ }^{13}$ Postimplantation syndrome can also occur with patients with stents or coil embolization. ${ }^{14}$ It constitutes a low-grade fever, abdominal pain, leukocytosis, and an elevation of the C-reactive protein. ${ }^{13}$ These can often be observed with conservative management in most cases. In extreme cases, blood flow can be reduced. If not enough collateral circulation exists, ischemia can occur in the colonic mucosa. ${ }^{15}$ Most of the existing literature regarding endovascular repair 
of iliac vessels is in the setting of atherosclerotic disease. Emergency stenting cases are rare and limited to isolated case reports. No definite set protocol exists for long-term follow-up of such patients. Further studies are warranted.

\section{CONCLUSION}

Penetrating abdominal injuries often cause significant vascular and nonvascular injuries that often require emergency surgery. Vascular injuries in a polytrauma patient are often associated with significant cardiovascular instability and solid organ injury. Wound contamination in acute scenarios, either due to visceral trauma or open wound, can make nonautologous repair of such injuries not an attractive option. Endovascular methods have become the standard of care in many institutions. The endovascular management of hemodynamically unstable patient involves the application of covered stent grafts. Such stent grafts are associated with decreased blood loss. It is a relatively less invasive procedure. Therefore, this requires less anesthesia and alleviates the need for elaborate dissection in a already traumatized field, as in our case. Such advantages can make a difference in critically injured trauma patients, especially in the geriatric population with multiple comorbidities. The fact is that endovascular approach can be performed from easily accessible sites, often remote from the injury sites, especially when identification and exposure of a vessel injury may be technically demanding.

\section{ACKNOWLEDGMENTS}

The authors acknowledge the trauma surgeons, physician assistants, ED physicians, and ICU nurses for their involvement in the clinical management of the patients, as well as the interventional radiology team for their prompt intervention.

\section{REFERENCES}

1. Gardiner MD, Mangwani J, Williams WW. Aneurysm of the common iliac artery presenting as a lumbosacral plexopathy. J Bone Joint Surg Br 2006 Nov;88(11):1524-1526.

2. Kirkwood ML. Surgical and endovascular repair of iliac artery aneurysm. UpToDate, 2014 Dec 19. Available from: www.uptodate.com/contents/surgical-and-endovascularrepair-of-iliac-artery-aneurysm.

3. Murphy EH, Woo EY. Endovascular today - endovascular management of common and internal iliac artery aneurysms. Endovascular Today Mar 2012.

4. Martin RS, Meredith JW. Management of acute trauma. In: Townsend CM, Beauchamp RD, Evers BM, Mattox KL, editors. Sabiston textbook of surgery: the biological basis of modern surgical practice. 19th ed. Philadelphia (PA): Elsevier Saunders; 2012. p. 455-457.

5. Baron BJ, Scalea TM, Sclafani SJ, Duncan AO, Trooskin SZ, Shapiro GM, Phillips TF, Goldstein AM, Atweh NA, Vieux EE, et al. Nonoperative management of blunt abdominal trauma: the role of sequential diagnostic peritoneal lavage, computed tomography, and angiography. Ann Emerg Med 1993 Oct;22(10):1556-1562.

6. McAnena OJ, Moore EE, Marx JA. Initial evaluation of the patient with blunt abdominal trauma. Surg Clin North Am 1990 Jun;70(3):495-515.

7. Weiss JJ. The importance of empiric abdominal computed tomography after urgent laparotomy for trauma: do they reveal unexpected injuries? National Center for Biotechnology Information, US National Library of Medicine.

8. Haste AK, Brewer BL, Steenburg SD. Diagnostic yield and clinical utility of abdominopelvic CT following emergent laparotomy for trauma. Radiology 2016 Sep;280(3):735-742.

9. Patel NV, Long GW, Cheema ZF, Rimar K, Brown OW, Shanley CJ. Open vs. endovascular repair of isolated iliac artery aneurysms: a 12-year experience. J Vasc Surg 2009 May;49(5):1147-1153.

10. Chaer RA, BarbatoJE, Lin SC, Zenati M, Kent KC, McKinseyJF. Isolated iliac artery aneurysms: a contemporary comparison of endovascular and open repair. J Vasc Surg 2008 Apr;47(4):708-713.

11. Sandhu RS, Pipinos II. Isolated iliac artery aneurysms. Semin Vasc Surg 2005 Dec;18(4):209-215.

12. Razavi MK, Dake MD, Semba CP, Nyman UR, Liddell RP. Percutaneous endoluminal placement of stent-graft for the treatment of isolated iliac artery aneurysms. Radiology 1995 Dec;197(3):801-804.

13. Sakamoto I. Endovascular treatment of iliac artery aneurysms. Radio Graphics 2005 Oct;25:S213-S227.

14. Velázquez OC, Carpenter JP, Baum RA, Barker CF, Golden M, Criado F, Pyeron A, Fairman RM. Perigraft air, fever, and leukocytosis after endovascular repair of abdominal aortic aneurysms. Am J Surg 1999 Sep;178(3):185-189.

15. Iliopoulos JI, Pierce GE, Hermreck AS, Haller CC, Thomas JH. Hemodynamics of the inferior mesenteric arterial circulation. J Vasc Surg 1990 Jan;11(1):120-126. 